\title{
Osteogenic Potency of Nacre on Human Mesenchymal Stem Cells
}

\author{
David W. Green ${ }^{1,4}$, Hyuk-Jae Kwon ${ }^{2,4}$, and Han-Sung Jung ${ }^{1,3, *}$
}

\begin{abstract}
Nacre seashell is a natural osteoinductive biomaterial with strong effects on osteoprogenitors, osteoblasts, and osteoclasts during bone tissue formation and morphogenesis. Although nacre has shown, in one study, to induce bridging of new bone across large non-union bone defects in 8 individual human patients, there have been no succeeding human surgical studies to confirm this outstanding potency. But the molecular mechanisms associated with nacre osteoinduction and the influence on bone marrow-derived mesenchymal stem cells (BMSC's), skeletal stem cells or bone marrow stromal cells remain elusive. In this study we highlight the phenotypic and biochemical effects of Pinctada maxima nacre chips and the global nacre soluble protein matrix (SPM) on primary human bone marrowderived stromal cells (hBMSCs) in vitro. In static co-culture with nacre chips, the hBMSCs secreted Alkaline phosphatase $(A L P)$ at levels that exceeded bone morphogenetic protein (rhBMP-2) treatment. Concentrated preparation of SPM applied to Stro-1 selected hBMSC's led to rapid ALP secretions, at concentrations exceeding the untreated controls even in osteogenic conditions. Within 21 days the same population of Stro-1 selected hBMSCs proliferated and secreted collagens I-IV, indicating the premature onset of an osteoblast phenotype. The same SPM was found to promote unselected hBMSC differentiation with osteocalcin detected at 7 days, and proliferation increased at 7 days in a dose-dependent manner. In conclusion, nacre particles and nacre SPM induced the early stages of human bone cell differentiation, indicating that they may be promising soluble factors with osteoinductive capacity in primary human bone cell progenitors such as, hBMSC's.
\end{abstract}

\footnotetext{
${ }^{1}$ Oral Biosciences, Faculty of Dentistry, The University of Hong Kong, Hong Kong SAR, ${ }^{2}$ Division of Developmental Biology, Cincinnati Children's Hospital Medical Center, Cincinnati, OH 45229, USA, ${ }^{3}$ Division in Anatomy and Developmental Biology, Department of Oral Biology, Oral Science Research Center, BK21 PLUS Project, Yonsei University College of Dentistry, Seoul, Korea, ${ }^{4}$ These authors contributed equally to this work.

*Correspondence: hsjung@hku.hk, hsjung@yuhs.ac
}

Received 14 November, 2014; accepted 20 November, 2014; published online 5 February, 2015

Keywords: bone morphogenetic protein, human bone marrow stromal cells, mesenchymal stem cells, nacre, nacre soluble matrix proteins, osteoinduction

\section{INTRODUCTION}

In an in vivo ectopic bone environment, a number of marine structures have shown osteoinductivity. This process involves the triggering of latent progenitors and pre-existing resident osteoblasts to conceive new bone from the beginning, de novo. Although there are many thousands of types of nacre seashell from different marine molluscs, pearl oyster (Pinctada maxima) nacre is the only species so far tested and validated for osteoinduction. Pearl oyster nacre is able to induce osteogenesis and bone formation from latent osteoprogenitors along an endochondral pathway, consisting of a cartilage tissue intermediary phase (Lamghari et al., 2001a; 2001b; Lopez et al., 1992; 2004a; 2004b; Milet et al., 2004; Shen et al., 2006; Silve et al., 1992). Nacre has been tested in human, sheep and rabbit models (Lamghari et al., 1999; 2001b; Liao et al., 2002). In human patients with critical size, non-union bone defects, fresh woven bone welded itself throughout the nacre implant with participation of osteoclasts and osteoblasts. It is important to have this cell partnership for continued bone health via remodeling. While nacre is stably tolerated in vivo, its degradation and resorption is limited and this could hinder its use within calcified tissue that requires rapid self-renewal (Lamghari et al., 2001a; 2001b). Naturally, this has steered the exploitation of nacre towards the true source of nacre osteoinductivity- the water-soluble organic matrix (WSM) fraction (Duplat et al., 2007; Rousseau et al., 2003). In this article we use the term nacre soluble protein matrix (SPM) in place of the established term (WSM) as it more accurately describes the entire protein extract that is removed from nacre seashell and used to influence hBMSC activities.

The SPM (acidic-rich particularly aspartic acid) participates in the control and regulation of crystallization and is an important stimulus on cell activities and intracellular molecular cascades associated with matrix mineralization. The strongest indications are that mobile peptides are released from the WSM when nacre is stripped from the shell and immersed in culture media or physiological fluids. The function of these mobile peptides mirrors that of transforming growth factor-beta (TGF- $\beta$ ) due to the correspondence in cell differentiation and proliferation timing profiles between bone marrow stromal cells (BMSCs) cultured with nacre and bone morphogenetic protein (BMP) and TGF- $\beta$-induced bone formation (Rousseau et al., 2003).

Decalcification of the calcium carbonate platelets and extraction of the WSM yields four identifiable fractions (Bédouet et al., 2006). Peptides are prevalent in the nacre matrix (Bédouet et al., 2007). Particular individual fractions have been shown to 
give rise to specific responses from cultured osteoblast cells. Protein fractions with low molecular weight (less than $1 \mathrm{kDa}$ ), for example, up-regulated alkaline phosphatase (ALP) secretion whereas high molecular weight fraction reduced ALP secretion.

Low molecular weight (LMW) fractions were recently found to increase gene expression of collagen type I, osteopontin, and Runx2 (Rousseau et al., 2008; Wang et al., 2007). Further detailed characterization of the bioactive LMW molecules has led to the identification of 110 molecules in the 100-700 Da range comprising of glycine-enriched peptides with structural similarities and high affinities for each other (Bédouet et al., 2006). p10, a highly defined matrix protein with a $10 \mathrm{kDa}$ size, has specifically demonstrated an induction of ALP expression in human fibroblast cell (Zhang et al., 2006), lending greater hope that the osteogenic signal molecules can be isolated in their vital functional form. A soluble p60 protein conglomerate extracted from decalcified nacre possesses sufficient bioactivity on MC3T3-E1 and MSCs to induce the secretion of mineral nodules (Lao et al., 2007). In another fractionation experiment, four proteins were found with homologous sequences to Wnt inhibitor factor-1 (WIF-1) and tenascin C, which are both involved in bone remodeling (Oliveira et al., 2012). The SPM has been found to upregulate osteoblast biomineralization and suppress osteoclast formation during bone remodelling and homeostasis. Recently it has been used to successfully repair osteoporotic bone in vivo (Kim et al., 2012).

In this study, we focus on the biochemical theme that has dominated nacre osteoinduction studies over more than a decade. Furthermore, for the first time, we have tested and evaluated nacre osteoinduction potency on primary human osteoprogenitors and mesenchymal stromal cells derived from the bone marrow. Our findings suggest that nacre based products possess the initial potential to "kickstart" bone regeneration from mesenchymal stem cell precursors. This evidence shows that the potency of nacre can be increased and provide new hope for treatment of patients.

\section{MATERIALS AND METHODS}

\section{Materials}

Cell culture media, dexamethasone, ALP kits, and all other biochemical reagents were of analytical grade and supplied by Sigma-Aldrich (USA) unless otherwise stated. Nacre wafers were obtained as offcuts from Gillett \& Son (UK).

\section{Production of nacre chips}

Nacre wafers previously sliced away from the shells of Pinctada maxima were ground and powdered in a ceramic pestle to generate an approximate $500-750 \mu \mathrm{m}$ dispersion of chip sizes using a fine test sieve (pore size $500 \mu \mathrm{m}$ ). These nacre chips were used in preference to a fine powder of nacre $(<100 \mu \mathrm{m})$, as used in some in vivo models, to prevent the removal of excessive volumes of deposited nacre during media changes on every alternate day. Due to their factory-based source the nacre chips were sterilized with bursts of $\gamma$-ray radiation at 2,500 rads every $12 \mathrm{~min}$ and ended after $5 \mathrm{~h}$. After washing in phosphate buffered saline (PBS), nacre chips were then thoroughly washed in antibiotic and anti-mycotic solution (Sigma-Aldrich, USA) at a $1 \times$ dilution for $24 \mathrm{~h}$ to kill any bacterial and fungal contaminants present in the nacre just prior to cell culture. The chips were then washed 3 times in PBS prior to human cell culture.

Primary human cell culture

Primary cultures of bone marrow cells were established from hematologically normal patients undergoing routine total hip replacement surgery. Samples were used with approval from the Southampton Hospital Ethics Committee (LREC 194/99). Human bone marrow cells were isolated as previously described by Yang et al. (2003). Bone marrow cells were maintained in 10\% $\alpha$ MEM at $37^{\circ} \mathrm{C}$ in a $5 \%$ humidified atmosphere. STRO- $1^{+}$enriched mesenchymal stem cell populations were isolated using the protocol as described by Stewart et al. (2003). The STRO-1 antibody hybridoma was kindly provided by Dr. J Beresford (University of Bath, UK). Preparation of STRO- $1^{+}$cell fractions was carried out as previously described using magnetic assisted cell sorting (MACS) (Yang et al., 2003).

\section{C2C12 pro-myoblast cell culture}

Mouse-derived pro-myoblast cells (C2C12) numbering $2 \times 10^{6}$ were cultured in Dulbecco's modified Eagle media (DMEM) supplemented with $10 \%$ FCS in the presence of $0.1 \mathrm{~g}$ of nacre chips (500-750 $\mu \mathrm{m}$ in size). In addition, C2C12 pro-myoblasts were cultured in DMEM containing $100 \mathrm{ng} / \mathrm{ml} \mathrm{rhBMP-2}$ to confirm the osteogenic conversion of these cells along with no treatment controls $(n=6)$. The cells were cultured for 5 days.

\section{Osteoinduction differentiation assay with nacre chips}

Following trypsinization from the culture flask and resuspension in serum-free $\alpha$-MEM, $4 \times 10^{5} \mathrm{C} 2 \mathrm{C} 12$ mouse promyoblast cells were seeded onto $0.25 \mathrm{~g}$ of fully sterilized nacre chips inside each 6-W-P culture well. The pro-myoblast seeded nacre chips were covered with basic media in one set of six wells and osteogenic media in another set of six wells. An identical number of $\mathrm{C} 2 \mathrm{C} 12$ pro-myoblast cells were also seeded into culture wells without the nacre chips again in basic and osteogenic media conditions, representing the control groups (n $=6$ ). All treatment and non-treatment groups of $\mathrm{C} 2 \mathrm{C} 12$ promyoblast cells were cultured on nacre for 3,7 and 14 days.

hBMSC viability and biocompatibility with nacre chips Live and necrotic osteoprogenitor cells were fluorescently tagged using Cell Tracker Green ${ }^{\mathrm{TM}}$ (CMFDA) (5-chloromethyl fluorescein diacetate) and Ethidium Homodimer-1 (EH-1) for 45 min in the incubator. CFMDA fluorescence is metabolically activated inside the cytoplasm of viable cells. The green viable cells and the red necrotic cells were detected using fluorescence microscopy with a Leica DMIRB/E (Leica Microsystems, Germany).

hBMSC growth and differentiation assays with nacre chips Primary unselected osteoprogenitor cell populations, numbering $2 \times 10^{6}$, at passage 1 , were seeded onto $0.25 \mathrm{~g}$ of nacre chips in 12 and 6 tissue culture well plates. Both cell types were cultivated for 14 days in basic media (supplemented with $10 \%$ FCS), osteogenic media (supplemented with 10\% FCS, dexamethasone and ascorbate) $(n=6)$, and media supplemented with $100 \mathrm{ng} / \mathrm{ml}$ of rhBMP-2 osteogenic growth factor. Concurrently, human osteoprogenitor cells were grown without nacre chips in basic, osteogenic, and rhBMP-2 infused media, representing the control groups $(n=6)$. Histological detection of ALP was performed following fixation in $95 \%(\mathrm{v} / \mathrm{v})$ ethanol using a Sigma ALP kit (Sigma-Aldrich, USA) according to the manufacturer's instructions. ALP enzyme activity was measured using p-nitro-phenyl phosphate as the substrate in 2-amino-2methyl-1-propanol alkaline buffer solution (1.5 M, pH 10.3 at $25^{\circ} \mathrm{C}$ ). The DNA content was measured using a PicoGreen molecular probe as per manufacturer's instructions (Molecular Probes, USA). The specific activity of osteoprogenitor cells was expressed as micro $(\mu)$ Moles of $p$-nitrophenol/h/ $\mu \mathrm{g}$ DNA +/-SD. 

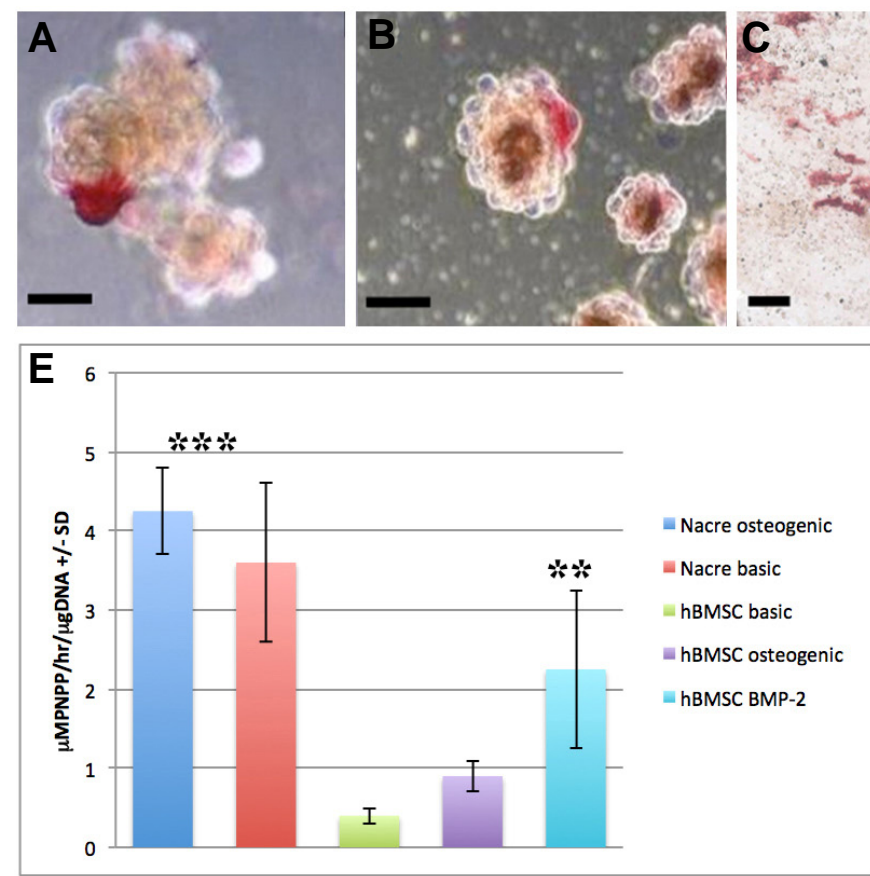
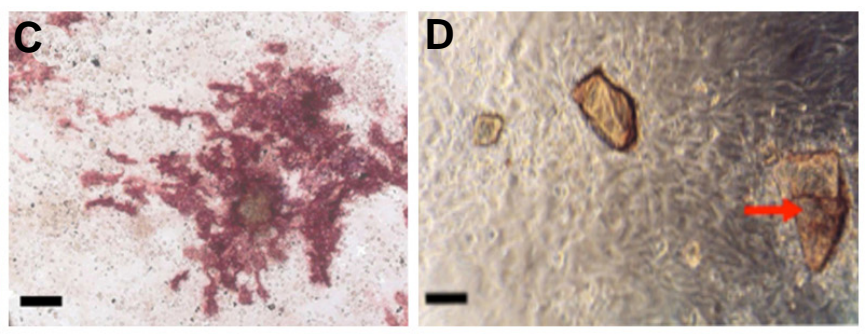

$\boldsymbol{F}$

Fig. 1. (A) $\mathrm{C} 2 \mathrm{C} 12$ pro-myoblast cell $3 \mathrm{D}$ pellet co-cultured with nacre particles in basic media and stained for $\mathrm{ALP}$ at day 7 (scale bar $=15 \mu \mathrm{m}$ ). (B) C2C12 pro-myoblast cell 3D pellet cultured in basic media supplemented with $100 \mathrm{ng} / \mathrm{ml}$ of rhBMP-2 and stained for ALP at day 7 (scale bar $=15 \mu \mathrm{m}$ ). (C) C2C12 pro-myoblast ALP expression in the presence of nacre chips at 14 days (scale bar $=25 \mu \mathrm{m})$. (D) Brightfield microscope image of human osteoprogenitors co-cultured with nacre chips (red arrow) at 7 days on tissue culture plastic (scale bar $=20 \mu \mathrm{m})$. (E) Human osteoprogenitor ALP activity with and without nacre at 7 days, in osteogenic and basic media compared with rhBMP-2 treatment (hBMSC basic media versus rhBMP-2, $\mathrm{p}<0.05$; hBMSC basic and osteogenic media with nacre versus HBMSC basic and osteogenic media without nacre, $p<0.01)$. (F) A table showing the variation in patient hBMSC ALP expression within and between Nacre and rhBMP-2 treated in vitro culture samples $(\mathrm{n}=3)$.

All ALP measurements were normalized against nacre alone negative control readings.

\section{Soluble matrix extraction from nacre particles (SPM)}

The WSM of nacre seashell was extracted following the EDTAbased method published by Almeida et al. (2001). This method was chosen in preference to a milder chemical-free extraction protocol using distilled water (Duplat et al., 2007). The reason was that the EDTA method yields organic matrix proteins closely bound and associated with calcium carbonate mineral where the water soaking method does not. And because of this, it yielded $25 \%$ more protein content for each extraction experiment. In brief, $0.5 \mathrm{~g}$ nacre particles were ground into a fine powder of particles approximately measuring $200 \mu \mathrm{m}$. The powder was then completely de-mineralized by soaking in $20 \mathrm{ml}$ of $1 \mathrm{M}$ EDTA disodium salt dihydrate while being continuously stirred for 14 days (Almeida et al., 2001). This aged EDTA solution contained SPM proteins dissolved away from the mineral framework of the particles. The solution was then dialyzed against pyrogen-free Milli-Q water (Millipore, USA) for 7 days at room temperature before use. This effectively removed all cytotoxic EDTA content. The concentration of extracted protein from treated nacre particles was measured using a Bradford protein detection assay kit (Bio-Rad Laboratories Inc., USA) according to the manufacturer's instructions. A low range of SPM concentrations were prepared, using the first direct extraction solution as a base. The further dilutions were made with pyrogen-free distilled water.

\section{Selected and unselected hBMSC cultivation with concentrated SPM or rhBMP-2}

STRO $-1^{+}$immunoselected hBMSCs were seeded $\left(2 \times 10^{4} / \mathrm{ml}\right)$ into culture wells of 6 well plates $(n=6)$ and covered with FCS free media comprising selected concentrations of SPM. The SPM concentration series was framed by the maximum yield of matrix proteins extracted using the EDTA method $(0.2 \mathrm{mg} / \mathrm{ml})$. In another osteogenic treatment group, $100 \mathrm{ng} / \mathrm{ml}$ of $\mathrm{rhBMP}-2$ was added to basic culture media with human mesenchymal stromal cells (hMSCs). The rhBMP-2 concentration applied was previously shown to induce the maximum osteogenic response in hBMSCs (Yang et al., 2003).

Assays for measuring osteosecretory activity of selected hMSCs with nacre SPM ALP activity

ALP activity was measured from nacre SPM and non-SPM treated selected hMSC populations as previously described.

\section{Sircol colourmetric binding assay for collagens I-IV} detection

Nacre SPM treated hBMSC samples were assayed for collagens (I-IV) using standard proprietary kits. Samples were dispersed in $300 \mu \mathrm{l}$ of $0.05 \%$ Triton, and cells were lysed by freeze-thawing three times. The Sircol Collagen Assay Kit (Biocolor Ltd., UK) was used to analyze collagen levels. Samples 

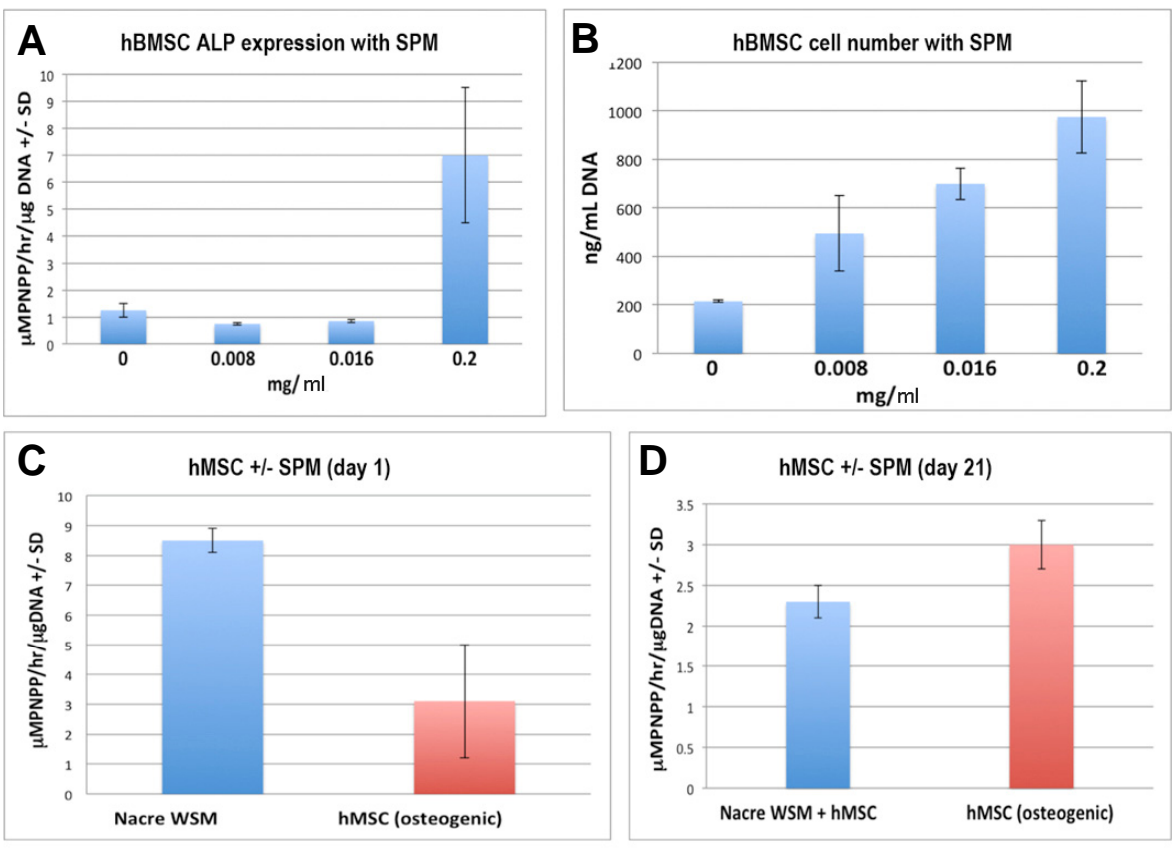

Fig. 2. (A) Dose-dependent action of extracted SPM on hBMSC ALP activity at day 3. (B) Dose-dependent action of extracted SPM on hBMSC number at day 28. (C) Comparison of ALP activity at $24 \mathrm{~h}$ between SPM-treated (180 $\mu \mathrm{g} / \mathrm{ml})$ immunoselected hMSC and hMSCs cultured in osteogenic media $(p<0.001, n=4)$. (D) Comparison of ALP activity at 21 days between SPM-treated $(180 \mu \mathrm{g} / \mathrm{ml}) \mathrm{im}$ muneselected hMSC and hMSCs cultured in osteogenic media ( $p<$ $0.01, n=4)$. (E) Quantitative collagen binding assay comparing immounoselected hMSCs treated with SPM and hMSCs without added SPM ( $p<$ 0.001, $n=4$ ).

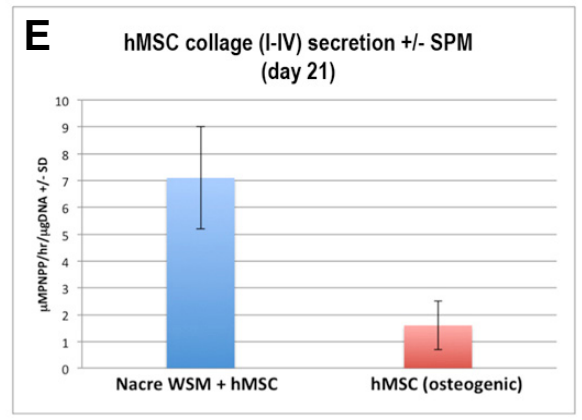

were run in triplicate and read at $540 \mathrm{~nm}$. Nacre only samples did not affect spectrophotometer readings.

\section{ELISA assay for osteocalcin protein detection}

hBMSC samples with and without SPM treatment were assayed for osteocalcin, a late marker for bone tissue formation. The samples were dispersed in $250 \mu \mathrm{l}$ of $0.05 \%$ Triton, and the cells were lysed by freeze-thawing three times. Osteocalcin was measured using the Intact Human Osteocalcin ELISA assay Kit according to the manufacturer's instructions (Biomedical Tech-nologies In., USA). Samples were run in triplicate and monitored at $540 \mathrm{~nm}$.

\section{Statistical analysis}

Statistical significance was analyzed using the unpaired t-test and ANOVA and all results are shown as mean \pm SD. Samples were run in triplicate for the biochemical assays. Statistical analysis was performed using GraphPad Instant Software (GraphPad Software Inc., USA). All experiments were repeated at least three times.

\section{RESULTS AND DISCUSSION}

Nacre osteoinduction differentiation assay with nacre chips The $\mathrm{C} 2 \mathrm{C} 12$ pro-myoblast cell line is exquisitely and specifically sensitive to rhBMP-2 osteogenic stimulation that results in differentiation along the osteoblast cell lineage. It has been used to test the osteoinductive capability of cells and proteins. To determine whether nacre induced a similar phenotypic response to rhBMP-2 treatment, $\mathrm{C} 2 \mathrm{C} 12$ pro-myoblast cells were co-cultured with nacre particles in vitro. The first result was that nacre chips were found to induce osteogenic differentiation in co-cultured pro-myoblasts as shown by strong positive ALP red staining in both traditional 2D (monolayer) and tissue-realistic 3D (pellet) cultures. This first occurred on day 2 (Figs. 1A and 2B). Nacre induced sporadic C2C12 ALP expression by day 7. Positive ALP expression of $\mathrm{C} 2 \mathrm{C} 12$ pro-myoblasts became much more extensive at 14 days reaching $70 \%$ of the total population of cells in monolayer culture and sphere cultures (Fig. 1C).

\section{Unselected primary hBMSC osteogenic responses to nacre particles in vitro}

Based on the finding that nacre particles could stimulate osteogenesis in pro-myoblasts, at potencies equivalent to rhBMP-2 osteoinductive stimulation, we extended the study to include primary human bone marrow derived cell populations (hBMSCs, BMSCs, skeletal stem cells). These cell types are now the foundation of current bone tissue engineering strategies in the 
laboratory context and gradually in clinical trials. Nacre particles with a fixed, uniform size range were cultured with unselected primary hBMSCs in static culture for up to 14 days (Fig. 1D). ALP activity in the presence of nacre chips was significantly increased compared to non-nacre controls at day 7 in both basic $(10 \%$ FCS) and ascorbate and dexamethasone supplemented osteogenic media (Fig. 1E; $p<0.01$ ). Thus, the nacre chips had the same specialization induction effect as osteogenic media. Importantly, the measured ALP response of unselected primary hBMSCs to osteogenic media and unselected primary hBMSCs treated with $100 \mathrm{ng} / \mathrm{ml}$ rhBMP-2 $(p<0.05)$ was significantly lower than nacre-treated unselected primary hBMSCs. Thus, hBMSCs co-cultured with nacre chips led to a stronger osteoinductive biochemical response than when they were treated to the most potent factor available for osteoinduction, rhBMP-2. It should be noted that these results represent an average of unselected primary hBMSCs from 3 different patients and it must be noted that there is significant variability between these different samples. There are other potential applications where a soluble and concentrated SPM product for osteoinduction is preferable.

\section{Effects of nacre SPM on selected primary hMSC and unselected primary hBMSC}

The SPM extract from nacre was added to serum-free culture media at concentrations varying between $0.008-0.2 \mathrm{mg} / \mathrm{ml}$ to test the dose-dependency of immuno-selected primary hMSC or unselected primary hBMSC populations. The highest concentration in this range generated the strongest early osteogenic response and so was the quantity used in the main experiments. Within 3 days the ALP activity of unselected primary hBMSCs was up-regulated when treated with 0.2 $\mathrm{mg} / \mathrm{ml}$ of SPM (Fig. 2A). The total cell number increased during this similar culture time, but significantly more so in cell populations treated with SPM. This response was uniformly dose-dependent in unselected hBMSC populations in a range of concentrations between 0 and $0.2 \mathrm{mg} / \mathrm{ml}$ (Fig. 2B). The lowest concentrations of SPM appear to have an effect on proliferation only. In 2D monolayer culture immuno-selected primary hMSC ALP expression was significantly elevated in the presence of $0.18 \mathrm{mg} / \mathrm{mL}$ SPM within $24 \mathrm{~h}$ (Figs. 2C and 2D; $\mathrm{p}<$ 0.001). However, at 7, 14, and 21 days, unselected hMSC ALP expression was significantly lower than the non-SPM treated controls. (Fig. 2D; $p<0.05$ ). Significantly, levels of bone associated collagens, as quantified by an ELISA assay were up-regulated in the SPM samples at 21 days indicating more advanced differentiation along the osteoblast lineage than control samples (Fig. 2E; $p<0.001$ ). It is noteworthy that, in unselected primary hBMSC populations, ALP secretion was absent at 14 days in culture indicating either unselected primary hBMSC had dedifferentiated or fully differentiated into bone forming precursor cells. Differentiation of unselected primary hBMSC into a committed osteoblast phenotype was confirmed at day 7 by measured secretion of osteocalcin $(3.5 \mathrm{ng} / \mathrm{ml}+/-2.9 ; \mathrm{n}=3)$. Osteocalcin was absent from all other nacre-free samples. The sequential pattern of rapid differentiation followed by proliferation observed in these nacre-treated samples also occurred in unselected primary hBMSC populations cultured in osteogenic media containing dexamethasone and ascorbate or rhBMP-2 at $100 \mathrm{ng} / \mathrm{ml}$ (data not shown).

Nacre is an osteoinductive nature derived biomaterial that invokes original new bone production from the beginning by activating human osteoblasts It has also sufficient potency to recruit and potentiate latent human osteoprogenitors to build new native, woven and lamellar bone in the mandible of a single human patient (Atlan et al., 1997). However, since that landmark human trial, there have been no previous follow-up studies to measure the first immediate osteoinductive responses of primary human progenitor cells, in cell culture and sourced from fresh bone marrow, to nacre chips and nacre SPM. Furthermore there are still questions over the osteoinductive authenticity of nacre. Since these cell types are now vital elements in tissue engineering of therapeutic bone replacements, it is important to test the osteoinductive responses of cell types with strong clinical relevance. We demonstrated in this study that nacre and its organic matrix directs the fate of co-cultured osteoprogenitor cells towards the osteoblast phenotype. We confirmed previous published results that the soluble protein-enriched and demineralized organic matrix of nacre is one of the most probable sources for this induction, although, we must not exclude the potential osteoinductive effects of the mineral structure on anchoring progenitors, which was highlighted in the nacre particle experiments. The fact that nacre chips can elicit such osteoinductive responses more potent than rhBMP-2 addition is very significant and also hints at nacre structure playing a role in the induction of cell differentiation. The use of nacre chips and organic matrix extracts typify the potency of nacre induced osteogenic differentiation of uncommitted and pre-committed precursors and raises expectation for a new accessible and abundant pre-made alternative to rhBMP-2. Nacre could potentially serve a role in therapeutic strategies for engineering clinically relevant original bone from the beginning with osteogenic precursor cells.

\section{ACKNOWLEDGMENTS}

This work was supported under the framework of international cooperation program managed by National Research Foundation of Korea (No. 2013K2A2A4003305).

\section{REFERENCES}

Almeida, M.J., Pereira, L., Milet, C., Haigle, J., Barbosa, M., and Lopez, E. (2001). Comparative effects of nacre water-soluble matrix and dexamethasone on the alkaline phosphatase activity of MRC-5 fibroblasts. J. Biomed. Mater. Res. 57, 306-312.

Atlan, G., Balmain, N., Berland, S., Vidal, B., and Lopez, E. (1997). Reconstruction of human maxillary defects with nacre powder: histological evidence for bone regeneration. C. R. Acad. Sci. III. 320, 253-258.

Bédouet, L., Rusconi, F., Rousseau, M., Duplat, D., Marie, A., Dubost, L., Le Ny, K., Berland, S., Péduzzi, J., and Lopez, E. (2006). Identification of low molecular weight molecules as new components of the nacre organic matrix. Comp. Biochem. Physiol. B Biochem. Mol. Biol. 144, 532-543.

Bédouet, L., Marie, A., Dubost, L., Peduzzi, J., Duplat, D., Berland, S., Puissegur, M., Boulzageut, H., Rousseau, M., Milet, C., et al. (2007). Proteomics analysis of the nacre soluble and insoluble proteins from the oyster Pinctada margaritifera. Mar. Biotechnol. 9, 638-649.

Duplat, D., Gallet, M., Berland, S., Marie, A., Dubost, L., Rousseau, M., Kamel, S., Milet, C., Brazier, M., Lopez, E., et al. (2007). The effect of molecules in mother-of-pearl on the decrease in bone resorption through the inhibition of osteoclast cathepsin K. Biomaterials 28, 4769-4778.

Kim, H., Lee, K., Ko, C.Y., Kim, H.S., Shin, H.I., Kim, T., Lee, S.H. and Jeong, D. (2012) The role of nacreous factors in preventing osteoporotic bone loss through both osteoblast activation and osteoclast inactivation. Biomaterials 33, 7489-7496.

Lamghari, M., Almeida, M.J., Berland, S., Huet, H., Laurent, A., Milet, C., and Lopez, E. (1999). Stimulation of bone marrow cells and bone formation by nacre: in vivo and in vitro studies. Bone 25, 91S-94S.

Lamghari, M., Antonietti, P., Berland, S., Laurent, A., and Lopez, E. (2001a). Arthrodesis of lumbar spine transverse processes us- 
ing nacre in rabbit. J. Bone Miner. Res. 16, 2232-2237.

Lamghari, M., Berland, S., Laurent, A., Huet H., and Lopez E. (2001b). Bone reactions to nacre injected percutaneously into the vertebrae of sheep. Biomaterials 22, 555-562.

Lao, Y., Zhang, X., Zhou, J., Su, W., Chen, R., Wang, Y., Zhou, W. and Xu, Z.F. (2007). Characterization and in vitro mineralization function of a soluble protein complex P60 from the nacre of Pinctada fucata. Comp. Biochem. Physiol. B Biochem. Mol. Biol. 148, 201-208

Liao, H., Mutvei, H., Hammarström, L., Wurtz, T., and Li, J. (2002). Tissue responses to nacreous implants in rat femur: an in situ hybridization and histochemical study. Biomaterials 23, 26932701.

Lopez, E., Vidal, B., Berland, S., Camprasse, S., Camprasse, G., and Silve, C. (1992). Demonstration of the capacity of nacre to induce bone formation by human osteoblasts maintained in vitro. Tissue Cell. 24, 667-679.

Lopez, E., Giraud, M., Le Faou, A., Berland, S., and Gutierrez, J. (1995). French Patent No FR 9515650, WO 97/24133-10/07/97.

Lopez, E., Antoinetti, P., Delattre, O., Milet, C., and Berland, S. (2004a). Nacre as a bone supply. Argos Spine News 8, 32-34

Lopez, E., Milet, C., Lamghari, M., Pereira-Mouries, L., Borzeix, S., and Berland, S. (2004b). The dualism of nacre. Key Eng. Mater 733, 254-256.

Milet, C., Berland, S., Lamghari, M., Mouriès, L., Jolly, C., and Borzeix, S. (2004). Conservation of signal molecules involved in biomineralization control in calcifying matrices of bone and shell. C. R. Palevol. 3, 493.

Oliveira, D.V., Silva, T.S., Cordeiro, O.D., Cavaco, S.I., and Simes, D.C. (2012). Identification of proteins with potential osteogenic activity present in the water-soluble matrix proteins from Cras sostrea gigas nacre using a proteomic approach. Scientific World Journal 2012, 765909.

Rousseau, M., Pereira-Mouriès, L., Almeida, M.J., Milet, C., and
Lopez, E. (2003). The water-soluble matrix fraction from the nacre of Pinctada maxima produces earlier mineralization of MC3T3-E1 mouse pre-osteoblasts. Comp. Biochem. Physiol. B Biochem. Mol. Biol. 135, 1-7.

Rousseau, M., Boulzaguet, H., Biagianti, J., Duplat, D., Milet, C Lopez, E., and Bédouet, L. (2008). Low molecular weight molecules of oyster nacre induce mineralization of the MC3T3-E1 cells. J. Biomed. Mater. Res. A 85, 487-497.

Shen, Y., Zhu, J., Zhang, H., and Zhao, F. (2006). In vitro osteogenic activity of pearl. Biomaterials 27, 281-287.

Silve, C., Lopez, E., Vidal, B., Smith, D.C., Camprasse, S. Camprasse, G., and Couly, G. (1992). Nacre initiates biomineralization by human osteoblasts maintained in vitro. Calcif. Tissue Int. 51, 363-369.

Stewart, K., Monk, P., Walsh, S., Jefferiss, C.M., Letchford, J., and Beresford, J.N. (2003). STRO-1, HOP-26 (CD63), CD49a and SB-10 (CD166) as markers of primitive human marrow stromal cells and their more differentiated progeny: a comparative investigation in vitro. Cell Tissue Res. 313, 281-290.

Wang, J.J., Chen, J.T., and Yang, C.L. (2007). Effects of soluble matrix of nacre on bone morphogenetic protein-2 and Cbfa1 gene expressions in rabbit marrow mesenchymal stem cells. Nan Fang Yi Ke Da Xue Xue Bao 27, 1838-1840.

Yang, X.B., Tare, R.S. Partridge, K.A.P., Roach, H.I. Clarke, N.M. Howdle, S.M., and Oreffo, R.O. (2003). Induction of human osteoprogenitor chemotaxis, proliferation, differentiation, and bone formation by osteoblast stimulating factor-1/pleiotrophin: osteoconductive biomimetic scaffolds for tissue engineering. $\mathrm{J}$. Bone Miner. Res. 18, 47-57.

Zhang, C., Li, S., Ma, Z., Xie, L., and Zhang, R. (2006). A novel matrix protein p10 from the nacre of pearl oyster (Pinctada fucata) and its effects on both $\mathrm{CaCO} 3$ crystal formation and mineralogenic cells. Mar. Biotechnol. 8, 624-633. 